\title{
Finstein, Poincaré e la Relatività speciale
}

\author{
di Emma Sallent del Colombo (Università di Barcellona)
}

Albert Einstein è di solito ritenuto I'unico autore della Teoria della relatività. A questa opinione sono state però mosse obiezioni in particolare per quanto riguarda i contributi di Henri Poincaré. La scarsità di testimonianze documentarie dirette sull'argomento e la mancanza di riferimenti incrociati fra i due autori, che pure si conobbero e si frequentarono ma che non si sono mai citati l'un l'altro, ha reso ancora più difficile fare luce su questo argomento. La controversia, basatasi spesso più su opinioni che su fatti, ha talvolta prodotto giudizi che hanno preso tinte nazionalistiche, antisemite, o dettate da esprit d'école.

Nel 1905 Albert Einstein pubblicò quattro fondamentali lavori uno sulla teoria quantistica, uno sul moto browniano e due di relatività, tra cui l'articolo considerato fondazionale della teoria speciale o ristretta: "Sull'elettrodinamica dei corpi in movimento".

Nel centenario del cosiddetto annus mirabilis einsteniano sono apparsi numerosi contributi tra i quali di particolare interesse è quello dello storico della Fisica francese Olivier Darrigol che, alla luce delle pubblicazioni degli ultimi 20 anni, fa una lettura profonda, critica ed equilibrata del binomio Einstein-Poincaré in "The Mistery of the Einstein-Poincaré Connection" (/sis, 2004). Secondo Darrigol, occorre abbandonare il pregiudizio di considerare i contributi di Einstein come a priori superiori a quelli dei suoi contemporanei che si sono occupati dell'argomento e valutare questi ultimi come giusti o sbagliati usando Einstein come unità di misura. Darrigol dimostra che è possibile intraprendere uno studio molto più interessante di come si possano spiegare le somiglianze e le differenze fra i diversi approcci abbandonando inutili e sterili polemiche di priorità.

Per poter mettere a confronto le teorie di Einstein e Poincaré occorre individuare lo strato concettuale che condividono leggendo i contributi dei due autori su ogni argomento nel loro contesto. Quali sono alcune delle idee che le due teorie hanno in comune?

L'analisi dei problemi legati all'elettrodinamica dei corpi in movimento conduce Einstein e Poincaré nel 1905 a postulare la validità del principio di relatività. Una formulazione comune ai due potrebbe essere che il risultato di ogni esperimento fisico concepibile è indipendente dal sistema inerziale di riferimento in cui si realizza. Tutti e due assumono che la velocità della luce è la stessa se misurata in diversi sistemi di riferimento inerziali e che lo spazio e il tempo misurati da osservatori appartenenti a diversi sistemi di riferimento inerziali sono in relazione tra di loro tramite le trasformazioni di Lorentz Igeneralizzazioni di quelle galileiane). Tutti e due riconoscono che le equazioni di Maxwell-Lorentz dell'elettrodinamica sono invarianti rispetto a queste trasformazioni; tutti e due richiedono infine che le leggi della Fisica siano invarianti rispetto alle trasformazioni di Lorentz e ricavano le leggi relativistiche del movimento.

Su alcuni punti, come il principio di relatività o l'interpretazione fisica delle trasformazioni di Lorentz, i contributi di Poincaré sono antecedenti a quelli di Einstein del 1905 di almeno 5 anni. Per il resto, i loro contributi sono praticamente simultanei. Poincaré pubblicò nel 1905 sui Comptes Rendues dell'Academie des Sciences una versione ridotta del suo "Sur la dynamique de l'electron" (che precede il lavoro di Einstein) e l'articolo in forma più estesa nel 1906 sui Rendiconti del Circolo Matematico di Palermo.

Quali le differenze concettuali? Einstein eliminò completamente l'etere dalla sua teoria, richiese che le leggi della Fisica fossero le stesse in ogni sistema di riferimento inerziale e introdusse una nuova cinematica in cui spazio e tempo nei diversi sistemi di riferimento inerziali giocano esattamente lo stesso ruolo. Poincaré mantenne invece l'etere come sistema di riferimento privilegiato nel quale sono definiti i "veri" spazio e tempo mentre spazio e tempo definiti in altri sistemi di riferimento sono solo "apparenti". Trattò la contrazione di Lorentz come un effetto derivato dal movimento di un regolo rigido attraverso l'etere mentre, per Einstein, è una conseguenza cinematica delle differenze tra le misure di spazio e tempo di osservatori inerziali diversi. Einstein derivò le trasformazioni di Lorentz dai suoi due postulati - principio di relatività e costanza della velocità della luce - mentre Poincaré derivò le trasformazioni come quelle che lasciano invarianti le equazioni di Maxwell-Lorentz dell'elettrodinamica. Sono diversità concettuali che non hanno in realtà conseguenze sperimentali per quanto riguarda l'Elettromagnetismo e l'Ottica. Come commentò Lorentz, la differenza è puramente epistemologica: ha a che fare con la quantità di elementi convenzionali ed arbitrari che si vogliono introdurre nelle definizioni dei concetti fisici di base.

Si tratta allora di un caso di scoperta simultanea? Stessi fattori e stessi interessi possono aver contribuito a simili studi oppure uno dei due - segnatamente Einstein - trovò in qualche modo ispirazione nelle opere dell'altro? Forse si possono dare risposte affermative ad entrambe le domande.

Einstein aveva letto Poincaré. Ma che cosa? Mentre non è accertato che Einstein conoscesse i contributi di Poincaré del 1905-6, sappiamo che lesse fra il 1902 e il 1905 La Science el'hypothèse. Con gli amici M. Solovine e C. Habicht fondò I'Akademie Olympia, un informale gruppo di lettura e discussione. Il libro li lasciò profondamente impressionati e senza fiato per varie settimane! Alcune delle idee che Einstein poté trovare nel libro di Poincaré sono lo scetticismo in relazione all'esistenza dell'etere, il principio di relatività, i problemi legati alla misura del tempo e la problematicità del concetto di simultaneità per due eventi che accadono in luoghi diversi. Sono tutte idee che compaiono poi nell'articolo di Einstein del 1905. Come propone Darrigol, sembra più saggio convenire che Lorentz, Poincaré ed Einstein abbiano tutti contribuito all'emergere della Teoria della relatività, che Poincaré ed Einstein abbiano offerto due diverse versioni della teoria e che Einstein abbia dato la forma che oggi è considerata migliore. A favorire l'affermarsi e consolidarsi del punto di vista di Einstein è poi intervenuta la prematura scomparsa di Poincaré, cui Einstein sopravvisse per ben 43 anni. 